\title{
硝基苯与离子液体的相互作用对硝基苯电还原传递系数的影响
}

\author{
陈 松 ${ }^{1,2}$ 马淳安 ${ }^{1, *}$ 褚有群 ${ }^{1}$ 毛信表 ${ }^{1}$ 柏云杉 $^{2}$ 陈 亮 ${ }^{2}$ \\ ( ${ }^{1}$ 浙江工业大学化学工程与材料学院, 绿色化学合成技术国家重点实验室培育基地, 杭州 310014; \\ 2 盐城工学院应用化学研究所, 江苏盐城 224002)
}

\begin{abstract}
摘要：应用紫外光谱研究了离子液体和硝基苯的相互作用, 硝基苯的紫外光谱受离子液体的影响, 硝基的吸收 峰红移, 末端吸收消失. 用循环伏安法研究硝基苯电还原过程中第 2 个电子转移的传递系数 $(\alpha)$ 随外界条件变化 所呈现复杂的变化规律. 硝基苯和水的介电性质、硝基苯和水与离子液体的相互作用对 $\alpha$ 产生复杂影响. 硝基苯 浓度增加使 $\alpha$ 减小, 离子液体的咪唑侧链增长也使 $\alpha$ 减小. 相同硝基苯浓度时, 水的浓度较低时, $\alpha$ 随水的浓度 增加变小; 水的浓度较高时, $\alpha$ 随水的浓度增加稍微变大. 温度升高 $\alpha$ 值增大.
\end{abstract}

关键词：紫外光谱; 硝基苯; $\mathrm{RMimBF}_{4}$; 电还原; 传递系数

中图分类号： O641; O646

\section{Effect of the Interaction between Nitrobenzene and Ionic Liquids on the Transfer Coefficient of the Nitrobenzene Electrochemical Reduction}

\author{
CHEN Song ${ }^{1,2} \quad$ MA Chun-An ${ }^{1, *} \quad$ CHU You-Qun ${ }^{1} \quad$ MAO Xin-Biao ${ }^{1}$ \\ BAI Yun-Shan ${ }^{2} \quad$ CHEN Liang ${ }^{2}$ \\ ('State Key Laboratory of Green Chemistry Synthesis Technology, College of Chemical Engineering and Materials Science, Zhejiang \\ University of Technology, Hangzhou 310014, P. R. China; ${ }^{2}$ Institute of Applied Chemistry, Yancheng Institute of Technology, \\ Yancheng 224002, Jiangsu Province, P. R. China)
}

\begin{abstract}
The interaction between ionic liquids (ILs) and nitrobenzene was investigated using ultraviolet spectroscopy. Compared to the ultraviolet spectrum in cyclohexane and water, a red shift of the absorption peak of the nitro group was observed in ionic liquids and no end absorption was found when the wavelength was less than $210 \mathrm{~nm}$. These phenomena may be attributed to the strong interaction between ILs and nitrobenzene. The transfer coefficient $(\alpha)$ of the electrochemical reduction of nitrobenzene for transferring the second electron in $\mathrm{RMimBF}_{4}$ (1-alkyl-3-methylimidazolium tetrafluoroborate) was investigated by cyclic voltammetry. The effect of the dielectric nature of nitrobenzene and water on the dielectric constants of the solution and on the capacitance of the electric double layer as well as the interaction between $\mathrm{EMimBF}_{4}$ (1-ethyl-3-methylimidazolium tetrafluoroborate) and nitrobenzene and water was found to have a complicated effect on $\alpha$. Increasing the concentration of nitrobenzene during the electrochemical reduction in $\mathrm{RMimBF}_{4}, \alpha$ decreased. Increasing the temperature the value of $\alpha$ increased while increasing the length of the imidazolium side chain had an opposite effect. At the same nitrobenzene concentration, with an increase in the water concentration $\alpha$ was reduced in $\mathrm{EMimBF}_{4}-\mathrm{H}_{2} \mathrm{O}$ system at lower water concentration and had reverse effect at higher water concentration.
\end{abstract}

Key Words : Ultraviolet spectroscopy; Nitrobenzene; $\mathrm{RMimBF}_{4} ; \quad$ Electrochemical reduction; Transfer coefficent

\footnotetext{
Received: March 30, 2009; Revised: June 22, 2009; Published on Web: August 19, 2009.

"Corresponding author. Email: science@zjut.cn; Tel: +86-571-88320011.

The project was supported by the Natural Science Foundation of Universities of Jiangsu Province, China (08KJD150007) and Project of State Key Laboratory of Green Chemistry Synthesis Technology, China (BK0107016).

江苏省高校自然科学基金(08KJD150007)及绿色化学合成技术国家重点实验室开放基金(BK0107016)资助项目
}

(C) Editorial office of Acta Physico-Chimica Sinica 
室温离子液体(RTILs)具有许多突出的优点, 因 其导电性好、电化学窗口宽、性质稳定, 作为特殊的 溶剂和电解质, 被广泛地应用于电化学研究领域 ${ }^{[1,2]}$. 由于对有机物的特殊溶解性以及有机物在离子液 体中特殊的反应性, 在有机电化学领域更引起人们 的广泛兴趣, 如: 离子液体中有机电还原 ${ }^{[3-6]}$ 、电氧 化 ${ }^{[7-9]}$ 、电聚合 ${ }^{[10-12]}$ 等反应. 硝基化合物电还原是有机 电化学领域的重要研究课题, 在离子液体中研究硝 基化合物的电还原行为受到人们更多的关注 ${ }^{[13-19]}$. Finklea ${ }^{[20]}$ 通过 Marcus 的态密度模型 ${ }^{[21]}$ 预测了单电 子转移过程和 1 电子 1 氢离子 $\left(1 \mathrm{e} 1 \mathrm{H}^{+}\right)$转移过程的传 递系数随过电位的变化规律, 并揭示了这种电位依 赖性质是氧化还原反应过程的重组能的函数. 陆 嘉星等 ${ }^{[199}$ 测定了离子液体中间硝基苯酚的传递系 数. 但是, 他们没有研究体系内外界因素对传递系数 的影响. 紫外光谱是研究溶剂与溶质相互作用的重 要手段 ${ }^{[2]}$. 本课题组在研究硝基苯和硝基甲烷在离子 液体中的电还原行为的基础上 ${ }^{[16-18]}$, 进一步通过紫 外光谱研究硝基苯与离子液体的相互作用, 探讨微 观电化学环境对硝基苯电还原第 2 个电子转移的 传递系数的影响, 对促进离子液体中硝基化合物电 还原的绿色化应用研究具有重要意义.

\section{1 实验部分}

\section{1 试剂与仪器}

硝基苯、环己烷、溴乙烷、1-溴丁烷、1-溴己烷、 $N$-甲基咪唑及四氟嗍酸铵, 均为 A.R. 级, 上海国药 集团提供; 二次去离子水, 自制. CHI660C 型电化学 工作站(美国辰华仪器公司), 核磁共振仪(VARIAN $400 \mathrm{MHz}, \mathrm{D}_{2} \mathrm{O}$ ), 紫外光谱仪(VARIAN CARY 100), 微量水测定仪(WA-1C, 江苏电分析仪器厂), 恒温水 浴(嘉兴市中新医疗有限公司).

\section{2 实验步骤}

采用文献[16,17]的方法制备离子液体 $\mathrm{RMimBF}_{4}$ (1-烷基- 3 -甲基四氟硼酸咪唑盐). $\mathrm{EMimBF}_{4}$ (1-乙 基-3-甲基四氟嗍酸咪唑盐)和 $\mathrm{BMimBF}_{4}$ (1-丁基3 -甲基四氟硼酸咪唑盐) 表征结果与文献相符, $\mathrm{HMimBF}_{4}$ (1-已基- 3-甲基四氟嗍酸咪唑盐)的核磁 共振谱为 ${ }^{~} \mathrm{H}$ NMR $\left(\mathrm{D}_{2} \mathrm{O}, \delta\right): 0.76\left(\mathrm{t}, \mathrm{CH}_{3}\right), 1.26(\mathrm{~m}$, $\left.\mathrm{CH}_{2}, 6 \mathrm{H}\right), 1.79\left(\mathrm{~m}, \mathrm{CH}_{2}\right), 3.87\left(\mathrm{~s}, \mathrm{~N}-\mathrm{CH}_{3}\right), 4.11(\mathrm{~m}, \mathrm{~N}-$ $\left.\mathrm{CH}_{2}\right), 7.43(\mathrm{~s}, \mathrm{NCH}), 7.48(\mathrm{~s}, \mathrm{NCH}), 8.52(\mathrm{~s}, \mathrm{NC}(\mathrm{H}) \mathrm{N})$. Karl-Fisher法 ${ }^{[23]}$ 测定微量水为 $0.03 \%$. 电化学测试同 文献[16]. 紫外光谱测试如下: 分别配制实验所需浓
度的硝基苯的环已烷、水和 $\mathrm{EMimBF}_{4}$ 溶液. 以 4 $\mathrm{nm} \cdot \mathrm{s}^{-1}$ 速率, 在 200-400 nm 范围内扫描得到相应的 图谱, 分别以环已烷、水和 $\mathrm{EMimBF}_{4}$ 为空白, 自动进 行背景扣除.

\section{2 结果与讨论}

\section{1 紫外吸收光谱研究硝基苯与 $\mathbf{E M i m B F}_{4}$ 的相 互作用}

硝基苯分子中有共轭结构, 苯基和硝基互为生 色和助色基团, 能够进行 $\pi \rightarrow \pi^{*}$ 和 $n \rightarrow \pi^{*}$ 的能级跃 迁产生紫外吸收光谱. 这种光谱不仅由分子本身的 结构决定, 还受到其所在介质环境的影响. 因此, 可 以通过测定溶液中硝基苯紫外光谱, 研究溶剂与硝 基苯的相互作用.

不同溶剂中的硝基苯紫外吸收光谱如图 1 所 示. 环已烷为紫外吸收惰性溶剂, 与硝基苯没有相互 作用, 在环已烷中硝基苯紫外吸收由苯环和硝基的 吸收带组成: 苯环有四个吸收谱带, 吸收最强的 $K$ 带 $\left(252 \mathrm{~nm}\right.$, 其摩尔吸光系数 $\varepsilon$ 约为 $\left.10000 \mathrm{~m}^{2} \cdot \mathrm{mol}^{-1}\right)$, 吸收弱的 $B$ 带 $\left(280 \mathrm{~nm}, \varepsilon\right.$ 约为 $\left.1000 \mathrm{~m}^{2} \cdot \mathrm{mol}^{-1}\right) 、 R$ 带 $\left(310 \mathrm{~nm}, \varepsilon\right.$ 约为 $\left.200 \mathrm{~m}^{2} \cdot \mathrm{mol}^{-1}\right) 、 E$ 带 $(<200 \mathrm{~nm}, \varepsilon$ 约 为 $200 \mathrm{~m}^{2} \cdot \mathrm{mol}^{-1}$ ), 通常出现 $K$ 带吸收峰, $B$ 带、 $R$ 带 被 $K$ 带覆盖, $E$ 带吸收不明显; 硝基有两个吸收谱 带, 在 $210 \mathrm{~nm}$ 附近出现 $\mathrm{N}=\mathrm{O}$ 双键的 $\pi \rightarrow \pi^{*}$ 跃迁 的强吸收峰, 该峰掩盖了苯环的 $E$ 带吸收, 在 270 $\mathrm{nm}$ 附近的 $\mathrm{N}=\mathrm{O}$ 孤对电子的 $n \rightarrow \pi$ *吸收很弱, 被苯 环的 $K$ 带吸收峰掩盖. 因此, 如图 la 所示测得的紫

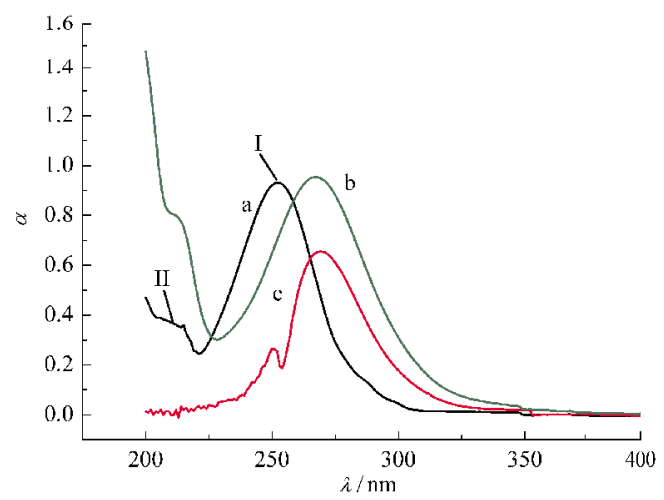

图 1 硝基苯在环己烷(a)、水(b)和 $\mathrm{EMimBF}_{4}$ (c)中的 紫外吸收光谱图

Fig.1 Ultraviolet absorption spectra of nitrobenzene in cyclohexane (a), water $(b)$, and $\mathrm{EMimBF}_{4}$ (c)

concentration of nitrobenzene $\left(c_{\mathrm{N}}\right): 1.0 \times 10^{-4} \mathrm{~mol} \cdot \mathrm{L}^{-1}$; $\mathrm{EMimBF}_{4}$ : 1-ethyl-3-methylimidazolium tetrafluoroboate 
外光谱图中可以看到 $252 \mathrm{~nm}$ (峰 I) 和 $210 \mathrm{~nm}$ (峰 II) 附近的两个吸收峰, 小于 $210 \mathrm{~nm}$ 的吸收峰在仪器 的测试范围之外. 作为对比, 测定了强极性分子溶剂 水中的紫外光谱, 如图 $1 \mathrm{~b}$ 所示, 水溶液中硝基苯在 $266 \mathrm{~nm}$ 处产生吸收峰, 较环已烷吸收峰红移; 与在 环已烷溶液中相比, 水溶液中硝基苯在 $210 \mathrm{~nm}$ 处 的吸收增大, $210 \mathrm{~nm}$ 以下的末端吸收也增大.

而在 $\mathrm{EMimBF}_{4}$ 中, 如图 1c 中硝基苯的紫外光 谱图形状出现了明显的变化, 在 $269 \mathrm{~nm}$ 产生最强 的吸收峰, 与水中相似; 在 $253 \mathrm{~nm}$ 处产生较弱的吸 收峰叠加在 $269 \mathrm{~nm}$ 的主峰上, 更为突出的是: 在 $210 \mathrm{~nm}$ 以下的末端吸收在扫描范围内消失. 由此可 见, $\mathrm{EMimBF}_{4}$ 中苯环的吸收变化不大, 而硝基的吸 收变化很大. 对此根据离子液体的特性进行一些探 讨. Bresme 等 ${ }^{[24]}$ 的研究发现因阴阳离子之间存在的 相互吸引作用, 使离子液体产生缔合而形成孔穴, 这 种孔穴可以容纳溶质分子. 这一研究结果有助于对 硝基的紫外吸收现象的理解. 硝基是硝基苯中的极 性基团, 离子液体形成的孔穴通过相互作用将硝基 苯包容进去, 硝基的 $\mathrm{N}=\mathrm{O}$ 双键因与咪唑阳离子相 互作用以及硝基 $\left(\mathrm{O}=\mathrm{N}^{+}-\mathrm{O}^{-}\right)$的 $\mathrm{N}$ 正离子与 $\mathrm{BF}_{4}^{-}$相 互作用, 均可以使 $\pi$ 轨道能量升高, $\pi *$ 轨道能量降 低, 能级差变小, 从而使紫外吸收峰红移. $210 \mathrm{~nm}$ 以 下吸收消失也与离子液体和硝基的相互作用密切相 关. 当然, 也可以用离子液体与溶质分子相互作用 的其它模型进行解释 ${ }^{[25]}$, 离子液体的咪唑阳离子可 以从硝基苯 $\mathrm{N}=\mathrm{O}$ 键的两面对其进行亲合作用, 或 硝基苯 $\mathrm{N}=\mathrm{O}$ 键从咪唑阳离子的环平面两侧对其进 行亲合作用, 同样可以产生上述紫外吸收现象. 由此 可见, 离子液体与硝基苯之间存在强烈的相互作用, 且主要作用在硝基和阴、阳离子之间.

\section{2 硝基苯在离子液体 $\mathbf{R M i m B F}_{4}$ 中的循环伏安} 行为

图 2 为硝基苯在离子液体 $\mathrm{RMimBF}_{4}$ 中的循环 伏安曲线, 在 $\mathrm{EMimBF}_{4} 、 \mathrm{BMimBF}_{4}$ 和 $\mathrm{HMimBF}_{4} 三$ 种离子液体中的行为相似. 由文献 $[16,17]$ 的研究结 果可知, 峰 I 的反应为第 1 个电子转移形成硝基苯 自由基阴离子, 是快速的电极过程; 峰 II 代表 2 个 电子的转移过程, 其中第 2 个电子转移后伴随质子 化-脱水过程生成亚硝基苯, 是慢速步骤, 第 3 个电 子转移形成的是亚硝基苯自由基阴离子, 是快速过 程, 并伴随快速的自由基偶合反应和质子化-脱水反 应, 生成氧化偶氮苯; 第 4 个电子转移也是慢速步

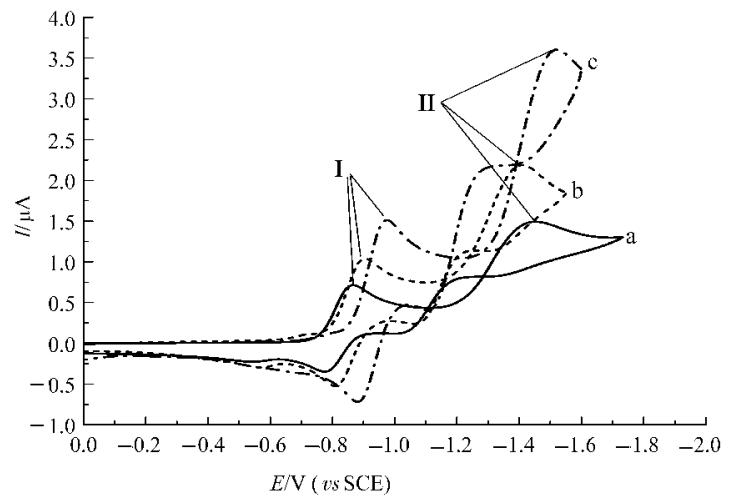

图 $2 \mathbf{R M i m B F}_{4}$ 中硝基苯的循环伏安图

Fig.2 Cyclic voltammograms of nitrobenzene in $\mathbf{R M i m B F}_{4}$

$\mathrm{Pt}, 298 \mathrm{~K}, c_{\mathrm{N}}=0.5 \mathrm{~mol} \cdot \mathrm{L}^{-1}$, scan rate $(v)=0.1 \mathrm{~V} \cdot \mathrm{s}^{-1}$; (a) $\mathrm{EMimBF}_{4}$ (b) $\mathrm{BMimBF}_{4}$ (1-butyl-3-methylimidazolium tetrafluoroboate),

(c) $\mathrm{HMimBF}_{4}$ (1-hexyl-3-methylimidazolium tetrafluoroborate)

骤, 与离子液体的还原相互影响. 因此, 第 2 个电子 转移是硝基苯还原的控制步骤. 该步骤的传递系数 是电极过程动力学的重要参数, 表征了过电位对该 步正逆反应活化能的影响. 紫外光谱的研究表明, 硝 基苯和离子液体所存在的上述作用, 必然对电极反 应的底物、过渡态和产物的状态和能级产生影响, 改 变电极溶液界面的电化学特性, 从而对该步骤传递 系数产生影响. 以下以该控制步骤为对象, 结合紫外 光谱的结果, 研究不同条件下电化学特性的变化对 动力学特征参数——传递系数的影响.

\section{3 硝基苯与离子液体的相互作用对传递系数的 影响}

硝基苯是电极反应的起始底物, 后续电极反应 的底物由它还原而产生. 为了考察硝基苯与离子液 体的相互作用对第 2 个电子转移的传递系数的影 响, 测定了不同硝基苯浓度下的循环伏安曲线. 结果 发现, 在 $\mathrm{EMimBF}_{4}$ 中硝基苯循环伏安曲线上峰 II 的半峰电位与峰电位之差 $\left(E_{\mathrm{hcII}}-E_{\mathrm{pcII}}\right)$ 随硝基苯浓度 增加而增大. 由不可逆过程的公式:

$$
E_{\mathrm{hcII}}-E_{\mathrm{pcII}}=1.857 R T / \alpha n_{\mathrm{a}} F
$$

计算得到传递系数 $\alpha$. 其中 $n_{\mathrm{a}}$ 为速度控制步骤转 移电子数 $1, T$ 为 $298 \mathrm{~K}, R$ 为气体常数, $F$ 为法拉第 常数, 结果列于表 1 (左边两列数据, $c_{\mathrm{N}}$ 为硝基苯浓度). 在纯离子液体中, 随硝基苯浓度增加, $\alpha$ 减小. 这说 明随着硝基苯浓度增加, 其获得第 2 个电子的电 极反应的活化能随过电位增加, 还原反应活化能减 小的趋势逐渐变小, 逆向氧化反应的活化能增加的 趋势变大.

由于溶液中硝基苯是非导电物质, 具有较大的 
表 $1 \mathrm{EMimBF}_{4}$ 中硝基苯的传递系数 $(\boldsymbol{\alpha})$

Table 1 Transfer coefficient $(\alpha)$ of nitrobenzene in $\mathbf{E M i m B F}_{4}$

\begin{tabular}{cccc}
\hline$c_{\mathrm{N}} /\left(\mathrm{mol} \cdot \mathrm{L}^{-1}\right)$ & $\alpha$ & $c_{\mathrm{W}} /\left(\mathrm{mol} \cdot \mathrm{L}^{-1}\right)$ & $\alpha^{*}$ \\
\hline 0.2 & 0.47 & 0.00 & 0.41 \\
0.5 & 0.41 & 1.67 & 0.25 \\
1.0 & 0.32 & 3.33 & 0.22 \\
2.0 & 0.30 & 5.55 & 0.23 \\
3.0 & 0.28 & 9.44 & 0.25 \\
4.0 & 0.26 & 16.67 & 0.26 \\
5.0 & 0.25 & & \\
\hline
\end{tabular}

$298 \mathrm{~K}, v=0.1 \mathrm{~V} \cdot \mathrm{s}^{-1} ; c_{\mathrm{w}}$ : concentration of water; ${ }^{*} c_{\mathrm{N}}=0.5 \mathrm{~mol} \cdot \mathrm{L}^{-1}$

介电常数 (36.4) ${ }^{[26]}$, 在电极与溶液形成的双电层区域, 其浓度增加使双电层的介电常数增大. 双电层电容 结构上类似平板电容, 平板电容器的电容公式 ${ }^{[27]}$ 为

$$
C=\varepsilon_{0} S / d
$$

其中, $S$ 为平板电容器的面积、 $d$ 为极板间的距离, $S$ 、 $d$ 与硝基苯浓度无关. $\varepsilon_{0}$ 为双电层的真空介电常数, 与硝基苯浓度有关. 由此可见, 随着硝基苯浓度的增 加, 双电层电容增大. 在相同过电位下, 硝基苯浓度 增加, 双电层电容储存电荷的能力大大提高 $(Q=$ $C U)$. 根据平板电容器的电势能计算公式 ${ }^{[27]}$ :

\section{$E=C U^{2} / 2=Q U / 2$}

其中, $Q$ 为双电层电容充放电的电量, $U$ 为双电层电 容的电压或过电位. 电容器储存电荷所消耗的电势 能随电容增大而增大. 在一定的过电位所产生的电 能传递给电化学体系, 当其中用于双电层充电的能 量增加时, 则用于电化学反应的能量就会减少, 必然 对动力学参数 (传递系数 $\alpha$ ) 产生影响. 因此, 传递系 数 $\alpha$ 随硝基苯浓度变化的微观本质是电极溶液界 面双电层性质的改变. 硝基苯与离子液体强烈的相 互作用对双电层性质产生复杂的影响, 使双电层的 结构发生变化, 传递系数 $\alpha$ 也随之出现复杂变化. 当硝基苯浓度较低时, 它能够较多地进人离子液体 形成的孔穴中, 随浓度增加, 双电层电容逐渐增大. 当硝基苯浓度较高时,一方面是孔穴已不足以容纳 增多的硝基苯, 同时逐渐增多的硝基与离子液体阴、 阳离子的强烈作用也会改变离子液体自身的缔合状 态, 阴、阳离子逐渐解除缔合, 溶液导电性增加, 而硝 基苯增加则使溶液介电常数增大, 两者共同作用, 使 硝基苯浓度大于 $1 \mathrm{~mol} \cdot \mathrm{L}^{-1}$ 时, 随其浓度增加, 双电 层变化渐渐变小而趋于恒定.

在双电层中进行的硝基苯电还原, 其第 2 个电 子转移的过程是:

$$
\mathrm{Ar}-\mathrm{NO}_{2}^{-\bullet}+\mathrm{e} \rightarrow \mathrm{Ar}-\mathrm{NO}_{2}^{2-}
$$

双电层电容越大, 越有利于储存负电荷, 负电性更高 的二价阴离子更易于被储存, 也即能量状态较一价 阴离子自由基更稳定, 电极反应的位能曲线的逆向 氧化反应的能垒高度相对还原反应的能垒高度变得 更大, 传递系数 $\alpha$ 就越小. 这就是硝基苯影响传递 系数 $\alpha$ 的途径和本质.

\section{4 水对传递系数的影响}

水与离子液体之间存在氢键作用 ${ }^{[25]}$, 同时为硝 基苯电还原的随后质子化反应提供质子, 因此, 研究 水的浓度变化对传递系数的影响具有重要意义. 当 固定硝基苯浓度而变化水的浓度时, 由式(1)计算的 $\alpha$ 值列于表 1 (右边两列, $c_{\mathrm{N}}$ 为 $0.5 \mathrm{~mol} \cdot \mathrm{L}^{-1}, c_{\mathrm{W}}$ 为水 的浓度), 结果发现, 随着水的浓度增加, $\alpha$ 先减小后 又稍稍增大. 由于水的介电常数很大 $\left(\varepsilon_{0}=78\right)^{[26]}$, 由 2.2 节的讨论可知, 水的加人会增大双电层电容. 水 的浓度低时, 水分子包容于离子液体的孔穴中, 只有 介电性质起作用, 使双电层电容增大. 当浓度较高 时, 由于水与离子液体的阴阳离子产生氢键作用, 改 变离子液体的缔合状态, 使之导电性增强以抵消水 的介电作用; 同时, 水给硝基苯自由基负离子提供 质子(见式(5)), 产生的氢氧根离子具有更强的导电 能力, 水的浓度越高, 自由状态的水越多, 硝基苯获 得第 2 个电子的反应方式就会更多地以式(5)进行, 这种作用就越大. 因此, 在水的浓度较高时, 随着水 浓度增加, 双电层电容又有所减小. 如 2.2 中所述的 双电层电容对氧化还原反应的能垒影响, 水对传递 系数产生上述的影响.

$$
\mathrm{Ar}-\mathrm{NOHO}+\mathrm{e}+\mathrm{H}_{2} \mathrm{O} \rightarrow \mathrm{Ar}-\mathrm{N}(\mathrm{OH})_{2}+\mathrm{OH}^{-}
$$

\section{5 $\mathrm{EMimBF}_{4}$ 中温度对传递系数的影响}

分别测定不同温度下 $0.5 \mathrm{~mol} \cdot \mathrm{L}^{-1}$ 硝基苯的循 环伏安曲线, 各曲线峰 II 的 $\left(E_{\mathrm{hcII}}-E_{\mathrm{pcII}}\right)$ 随温度升高而 减小. 由式(1)计算的结果可知, 温度为 298 、318、338 和 $358 \mathrm{~K}$ 时, 对应的 $\alpha$ 值分别为 $0.41 、 0.51 、 0.71$ 和 $0.82, \alpha$ 值随温度升高而增大. 由介电物质的介电常 数的温度特性可知, 随温度升高, 硝基苯的介电常数 迅速变小, 双电层电容减小, 使 $\alpha$ 值增大. 同时, 温 度升高, 离子液体的阴、阳离子能量增大, 能够克服 离子间静电作用而使缔合程度降低, 导电性增加, 也 造成双电层电容减小, 使 $\alpha$ 值增大.

对上述 $\alpha$ 与 $T$ 进行曲线拟合, 得到图 3 所示的 结果, 其相互关系为 $\alpha=-1.73+0.0072 T$ (相关系数 $r=$ 0.992), 可见 $\alpha$ 与 $T$ 存在较好的线性关系, 与文献 


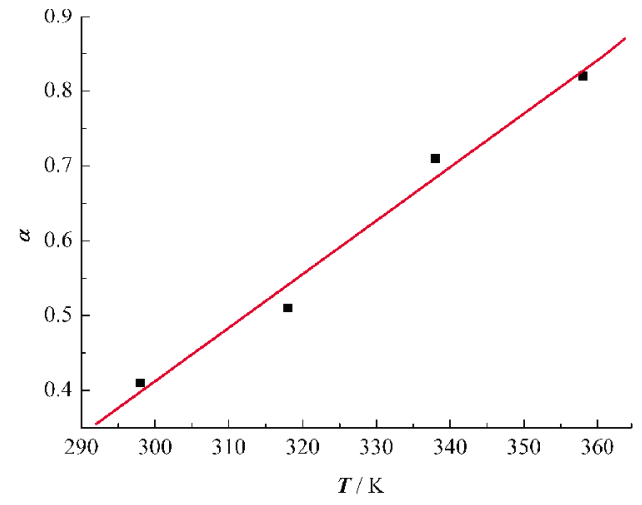

图 3 传递系数 $\boldsymbol{\alpha}$ 和温度的关系

Fig.3 Plot of the relationship between transfer coefficient $\alpha$ and temperature

表 2 不同离子液体中硝基苯的传递系数

Table 2 Transfer coefficient of nitrobenzene in different ionic liquids

\begin{tabular}{cccc}
\hline \multirow{2}{*}{$c_{\mathrm{N}} /\left(\mathrm{mol} \cdot \mathrm{L}^{-1}\right)$} & \multicolumn{3}{c}{$\alpha$} \\
\cline { 2 - 4 } & $\mathrm{EMimBF}_{4}$ & $\mathrm{BMimBF}_{4}$ & $\mathrm{HMimBF}_{4}$ \\
\hline 0.2 & 0.47 & 0.46 & 0.39 \\
0.5 & 0.41 & 0.40 & 0.33 \\
1.0 & 0.32 & 0.32 & 0.24 \\
2.0 & 0.30 & 0.23 & 0.20 \\
3.0 & 0.28 & 0.20 & 0.18 \\
\hline
\end{tabular}

[28]的结果相符合, 这就意味着电极电位对反应速 度的影响主要通过活化熵的变化, 而电极电位影响 活化熵的途径之一就是改变双电层结构(也即改变 双电层电容), 这也与本文 $2.2 、 2.3$ 节及本节前面部 分的分析相吻合.

\section{6 不同离子液体对传递系数的影响}

为了考察硝基苯与不同离子液体的相互作用对 传递系数的影响, 测定了咪唑阳离子侧链不同的离 子液体 $\mathrm{BMimBF}_{4} 、 \mathrm{HMimBF}_{4}$ 中硝基苯的循环伏安 曲线, 结果发现: 与 $\mathrm{EMimBF}_{4}$ 中相似, 在这两种离 子液体中, 硝基苯循环伏安曲线的 $\left(E_{\mathrm{hcII}}-E_{\mathrm{pcII}}\right)$ 均随浓 度增加而增大. 根据式(1)计算结果列于表 2. 随着浓 度增加, 三种离子液体中的 $\alpha$ 值均减小; 硝基苯浓 度相同时, 在三种离子液体中的 $\alpha$ 值大小顺序为 $\mathrm{EMimBF}_{4}>\mathrm{BMimBF}_{4}>\mathrm{HMimBF}_{4}$. 这是由于三种离 子液体中咪唑阳离子侧链依次增长, 导电性依次降 低, 相同硝基苯浓度时, 双电层介电常数依次增大, 使 $\alpha$ 值依次减小.

\section{3 结 论}

硝基苯在离子液体中的紫外光谱, 硝基吸收峰
红移, 末端吸收消失, 表明离子液体 $\mathrm{EMimBF}_{4}$ 对硝 基苯的作用点主要在硝基上. 受此影响, 硝基苯电还 原的第 2 个电子转移的传递系数 $\alpha$ 随外界条件变 化呈现一定规律: 硝基苯和水的介电性质, 双电层 电容变化, 引起 $\alpha$ 随之变化; 随着硝基苯浓度增加, $\alpha$ 减小, 离子液体咪唑侧链增长也使 $\alpha$ 减小; 离子液 体与硝基苯以及水的相互作用, 使 $\alpha$ 出现复杂的变 化; 通过升高温度可以提高 $\alpha$ 值.

\section{References}

1 Buzzeo, M. C.; Evans, R. G.; Compton, R. G. ChemPhysChem, 2004, 5: 1106

2 Robonson, J.; Osteryoung, R. A. J. Am. Chem. Soc., 1979, 102: 323

3 Mellah, M.; Gmouh, S.; Vaultier, M.; Jouikov, V. Electrochem. Commun., 2003, 5: 591

4 Barhdadi, R.; Courtinard, C.; Nedelec, J. Chem. Commun., 2003: 1434

5 Doherty, A. P.; Brooks, C. A. Electrochim. Acta, 2004, 49: 3821

6 Zhao, G. Y.; Jiang, T.; Han, B. X.; Li, Z. H.; Zhang, J. M.; Liu, Z. M.; He, J.; Wu, W. Z. J. Supercrit. Fluids, 2004, 32: 287

7 Martiz, B.; Keyrouz, R.; Gmouh, S. Chem. Commun., 2004: 674

8 Hasegawa, M.; Fuchigami, T. Electrochim. Acta, 2004, 49: 3367

9 Villagrán, C.; Aldous, L.; Lagunas, M. C.; Compton, R. G.; Hardacre, C. J. Electroanal. Chem., 2006, 588: 27

10 Sekiguchi, K.; Atobe, M.; Fuchigami, T. J. Electroanal. Chem., 2003, 557: 1

11 Sekiguchi, K.; Atobe, M.; Fuchigami, T. Electrochem. Commun., 2004, 4: 881

12 Li, M. C.; Ma, C. A.; Liu, B. Electrochem. Commun., 2005, 2: 209

13 Silvester, D. S.; Wain, A. J.; Aldous, L.; Hardacre, C.; Compton, R. G. J. Electroanal. Chem., 2006, 596: 131

14 Corinne, L.; Loredana, P.; Elena, V.; Philippe, H. J. Electroanal. Chem., 2005, 585: 1

15 Liu, Y. Z.; Xiao, L. P.; Zhang, K.; Zhao, S. F.; Zhang, J. B.; Lu, J. X. Chem. J. Chin. Univ., 2008, 29: 2059 [柳英姿, 肖丽平, 张 凯, 赵淑凤, 张静波, 陆嘉星. 高等学校化学学报, 2008, 29: 2059]

16 Chen, S.; Ma, C. A.; Chu, Y. Q.; Mao, X. B. Chem. J. Chin. Univ., 2007, 28: 1935 [陈 松, 马淳安, 褚有群, 毛信表. 高等学校化 学学报, 2007, 28: 1935]

17 Ma, C. A.; Chen, S.; Chu, Y. Q.; Mao, X. B. Acta Phys. -Chim. Sin., 2007, 23: 575 [马淳安, 陈 松, 褚有群, 毛信表. 物理化 学学报, 2007, 23: 575]

18 Ma, C. A.; Wang, X. J.; Li, G. H.; Li, M. C.; Chen, S. Journal of Chemical Industry and Engineering, 2008, 59: 653 [马淳安, 王晓娟, 李国华, 李美超, 陈 松. 化工学报, 2008, 59: 653]

19 Wang, H.; Ye, X. H.; Chen, L. M.; Lu, J. X.; He, M. Y. Chem. J. Chin. Univ., 2005, 26: 326 [王＼cjkstart欢, 叶小鹤, 陈黎明, 陆嘉星, 
何敏元. 高等学校化学学报, 2005, 26: 326]

20 Finklea, H. O. J. Electroanal.Chem., 2001, 495: 79

21 Marcus, R. A. J. Chem. Phys., 1965, 43: 679

22 Wang, P. H.; Zhang, Y. F.; Zhang, Q.; Chen, S. W.; Yuan, W. B.; Wu, S. S. Spectroscopy and Spectral Analysis, 2007, 27: 2250 [王平红, 张寅峰, 张 岐, 陈尚文, 袁文兵, 吴姗姗. 光谱学与光 谱分析, 2007, 27: 2250]

23 Zhu, J. Y. Organic analysis. Beijing: Chemical Industry Press, 2004: 171 [朱嘉云. 有机分析. 北京: 化学工业出版社, 2004: 171]

24 Bresme, F.; Alejandre, J. J. Chem. Phys., 2003, 118: 4134
25 Cammarata, L.; Kazarian, S. G.; Salterb, P. A.; Weltonb, T. Phys. Chem. Chem. Phys., 2001, 3: 5192

26 Zhu, Y. B. Electrochemical data handbook. Changsha: Hunan Science and Technology Press, 1985: 596, 569 [朱元保. 电化学 数据手册. 长沙: 湖南科学技术出版社, 1985: 596, 569]

27 Cheng, S. Z.; Jiang, Z. Y. General physics. Beijing: Higher Education Press, 1998: 98, 137 [程守洙, 江之永. 普通物理学. 北京: 高等教育出版社, 1998: 98, 137]

$28 \mathrm{Wu}, \mathrm{H}$. H. Electrochemistry. Beijing: Chemical Industry Press, 2004: 84 [吴辉煌. 电化学. 北京: 化学工业出版社, 2004: 84] 